\title{
The Random Design Argument
}

\author{
An Example of Science Under Theological Influence
}

\author{
Cornelius Hunter \\ DOI: 10.33014/issn.2640-5652.1.2.hunter.1
}

\begin{abstract}
The "random design argument" is a popular mode of justification, used to prove that scientific theories are true. The first part of this argument is that, if God had designed X, the features of $\mathrm{X}$ would approximate randomness. The second part of this argument is that random design can therefore be used, in the form of a null hypothesis, as a proxy for design. If the null hypothesis can be falsified, then design is falsified and contingency is confirmed. The random design argument has two important problems that have gone unrecognized. First, though presented as a finding of empirical science, it relies on theological claims. Second, though presented as a null hypothesis, there is no justification that random design is the logical complement of the scientific theory in question. Despite its failings, the random design argument has a long history of use for justifying and proving scientific hypotheses. This paper documents several areas where the random design argument has been used (both currently and historically) as well as the logical problems with the argument.
\end{abstract}

\section{Introduction}

In his book The Evidence for Evolution, Alan Rogers employs contrastive reasoning to argue that evolutionary theory is far superior to creationism. Regarding the universal genetic code, he explains that as with human language, the genetic code is arbitrary. Nothing forces us, for example, to use the word "bird" for animals with wings and feathers. Rogers concludes: "Different species could have different genetic codes just as easily as different human populations speak different languages. But they don't. Every living thing - from the smallest microorganism to the largest whale - makes protein using essentially the same arbitrary code. What sense does this make, unless all these forms of life evolved from a single ancestor?" (Rogers, 2011, pg. 31) The two key premises in Rogers' argument are that the genetic code is arbitrary, and that it is universal. In other words, the code's design space is unconstrained (most any code would work), but in practice the code is highly constrained (all species have essentially the same code). There is a stark contrast between the potential and the actual. The different species "could have different genetic codes ... [b]ut they don't." This for Rogers is powerful evidence against independent creation and for evolution. But why? Superficially this appears to be a weak argument.

Rogers' argument appears to amount to the following logical flow. If the species evolved, then it is plausible, or likely, that the genetic code is identical or highly similar across the species. But if the species were independently created, then the genetic code could vary across the species. An interlocutor would be delighted. Clearly this argument does not rule out creationism. The argument does not even show that creationism is unlikely. Yes, God could create different genetic codes in the different species, but we could just as easily state that God could create the same genetic codes in the different species. Superficially, the argument does not seem to serve Rogers' purpose. The argument seems to be at best, trivial, and at worst, invalid, for the conclusionthat the evidence makes no sense except on evolution - does not follow from the premises.

To make matters worse, the above example from Rogers is not a rare exception. This argument - that the finding of a single characteristic in an otherwise unconstrained design space, where many alternative designs are possible, is evidence for evolution - is not uncommon (as we shall see in the following sections). Furthermore, not only is this argument not uncommon, it is presented as powerful and decisive. Have evolutionists made a terrible mistake in committing to, and promoting, an invalid argument?

In this paper I demonstrate this is not the case. This evolutionary argument, as exemplified by Rogers above, can be seen to be perfectly valid once the context is understood. The key to Rogers' argument is the underlying evolutionary premise that if the species were independently created, then their designs would fill the design space. The potential should be realized in the actual. There should be no gratuitous repetitions of a design. Instead, designs should be scattered throughout the design space, leaving no dis- 
cernable gaps or patterns.

Exceptions can arise only if the design space is functionally constrained. That is, if only certain designs will work then, of course, some level of repetition will be required. That is why it is significant for Rogers that the "[d] ifferent species could have different genetic codes." [emphasis added] In other words, the design space is not constrained, and so under independent creation there is no reason why the species should not have varied genetic codes. But they do, and so contingency is implicated. Mark Ridley makes the argument more explicit in his Evolution textbook. After explaining that the code is unconstrained, he concludes that "[t]he universality of the genetic code is important evidence that all life shares a single origin." For it is "similar between species, but would not be if the species had independent origins." (Ridley, 1993, pgs. 48-49)

Here Ridley makes explicit the expectation for independently created species. They must fill the design space. Clearly this argument entails non empirical content. The origins and details of the underlying metaphysics are beyond the scope of this paper. I will merely refer to the argument as the "random design argument." (following Hunter, 2007 \& Hunter, 2014)

The form of the random design argument is as follows:

1. God would create the fullest creation without gaps.

2. We observe arbitrary or idiosyncratic patterns which do not fill the full range of possibilities. That is, they do not fill the naturally available design space.

3. Such patterns would not have been divinely intended, and so must have arisen via contingent events.

Step 1 states that a divinely designed world would have no unnecessary gaps. Therefore, such a world would not be limited to a particular, single, design where multiple designs are possible. A divine design would explore the entire range. Step 2 acknowledges that we find limitations in nature's designs which do not seem to be necessary. That is, there are designs that could exist which do not exist. This suggests that nature has gaps, and its designs are arbitrary. Step 3 concludes that this falsifies divine design, and therefore is strong evidence for contingency.

Evolutionists often express this argument qualitatively, as exemplified by Rogers and Ridley above. But it also lends itself conveniently to a powerful quantitative form: null hypothesis testing. If it could be shown that random design is false, then contingency could be declared to be true. As we shall see in Section 2, mathematical techniques were developed in the early eighteenth century to perform this type of test. In the twentieth century, the modern discipline of statistics formalized null hypothesis testing. (Fisher, 1935) The null hypothesis is the opposite of the hypothesis in question. That is, the null hypothesis is what is expected if the hypothesis in question, referred to as the alternate hypothesis, is false. If the null hypothesis can be shown to be statistically false, then the alternate hypothesis is declared true.

In this simple form, the null hypothesis is rejected if its probability, computed as the so-called $P$ value, is sufficiently small. When the null hypothesis is rejected, then the alternate hypothesis is accepted. This simple null hypothesis test presents a dichotomy, and for it to function properly it is crucial that no other explanations are possible. That is, there must be only one alternate explanation. Otherwise, the test presents a false dichotomy.

Twentieth century null hypothesis testing formalized the earlier mathematical techniques developed in the eighteenth century (though I know of no connection between the two). As we shall see, the development of null hypothesis testing further bolstered this approach of statistically falsifying a model of what we expect of nature under random design.

We will see in Sections 2 and 3 that random design became the null hypothesis in cosmology and biology, respectively, even before such statistical tests were formalized in the early twentieth century. The random design model was represented by hypothetical designs that filled the design space and had no gaps. The design space should be full, with the designs appearing not at merely a few locations within the space, but rather throughout the space, at random. As we will see, the random design null hypothesis test was, and remains today, an extremely powerful and influential argument for contingency. Today, it is a fixture of evolutionary reasoning that is common across numerous academic authors. Finally, Section 4 provides the conclusions of this study.

\section{The influence of the random design argument in cosmological evolution}

Isaac Newton explained the solar system with his new physics and law of universal gravitation. The planets revolved about the sun, and the moons about their respective planets, according to the gravitational force and laws of motion. It was a grand unification of what had been 
Aristotle's two, very different, sub and superlunar regions. This new view of the cosmos was simple and stunning, and Newton went on to make a series of arguments for why the solar system exhibits design rather than a naturalistic origin. First, Newton argued that while his laws explained the operation of the solar system, they could not explain the origin of the solar system:

the planets and comets will constantly pursue their revolutions in orbits given in kind and position, according to the laws above explained; but though these bodies may, indeed, persevere in their orbits by the mere laws of gravity, yet they could by no means have at first derived the regular position of the orbits themselves from those laws. (Newton, 1687, pg. 440)

Newton also made an argument from what we might call fine-tuning. In order for the planets to fall into their concentric orbits about the sun, their velocities would need to fall into certain ranges, as he explained in a letter to Richard Bentley in 1692:

Nor is there any natural cause which could give the planets those just degrees of velocity, in proportion to their distances from the sun and other central bodies, which were requisite to make them move in such concentric orbs about those bodies. (Danielson, 2000, pg. 228)

Finally, Newton repeatedly argued that the patterns found in the solar system revealed design. Specifically, while the comets exhibited random orbits, the planets and their moons exhibited consistent patterns. If the comets revealed what could be expected from the action of blind natural processes, the planets revealed a stark contrast of consistency.

For while comets move in very excentrick orbs in all manner of positions, blind Fate could never make all the planets move one and the same way in orbs concentrick, (Newton, 1718)

As William Derham later reported, Newton specifically noted that the planets revolve about the sun in the same direction, and that "their Orbits have all the same inclination." (Manuel, 1968, pg. 127) Newton was, of course, well aware that the planetary orbits did not share the exact same inclination angle. But they are sufficiently similar to form, at least roughly, a plane in which the planets travel as they orbit the sun. In comparison with the comets, whose orbits were at seemingly random inclination angles and in no relation to each other, the planets exhibited a striking pattern and similarity.

But for what purpose was this pattern? Newton's argument that the solar system patterns revealed common design could be turned on its head. For the patterns could just as easily be seen as the result of a common mechanism. This was the view of mathematician and scientist Daniel Bernoulli in his award-winning 1734 essay on the origin of the solar system at the Paris Academy. Bernoulli contrasted the inclination angles of the planets with a random design where the planetary orbits were inclined at random angles. If the planetary orbits had fallen into place by chance, it would be highly improbable that they would just happen to lie in practically the same plane. Bernoulli gave three different calculations, all of which showed the odds were astronomical. He picked the middle result of the three, which was that the odds of such a coincidental alignment are $1,419,856$ to 1 . Bernoulli arrived at this value by dividing the range of possible orbital inclination angles into 17 bins. The orbital inclination angles of the six known planets all fell into the same bin. If the planetary orbits were inclined at random angles, the chances of them all falling into the same bin would be 1 in $17^{6-1}$, or 1 in $1,419,856$. This is highly improbable so Bernoulli argued that the sun's atmosphere was the cause of the alignment of the planetary orbits. He who would deny this, concluded Bernoulli, "must reject all the truths, which we know by induction." (Bernoulli, 1734)

Bernoulli's 1734 paper is an example of what today would be referred to as null hypothesis testing, long before Fisher's formalization of this method in the twentieth century. The null hypothesis, in this case, states that the planetary orbits, and in particular their inclination angles, are randomly distributed over the space. The striking consistency of the planetary orbits is highly unlikely on this null hypothesis. Therefore, Bernoulli rejected it, and concluded his alternate hypothesis must be true, that the orbits were formed by the sun's atmosphere.

Bernoulli's paper was an early example of the random design argument. Immanuel Kant used similar reasoning twenty years later in his ambitious and expansive cosmological treatise. ${ }^{1}$ Kant's approach was a blend of mechanical principles and metaphysics. (Shea, 1986, pg. 119) Regarding the solar system, Kant's random design argument now added ten moons in addition to the six planets, and

\footnotetext{
${ }^{1}$ Kant did not mention Bernoulli, but he did make use of Bernoulli's 17 divisions of inclination angle in discussing the volume of space that would contribute matter to the forming planets. (Kant, 1755, pgs. 53$56)$
} 
included additional orbital parameters: the axial rotation, and revolution about the sun, in addition to the orbital inclination angle.

These movements have a continuous shared direction: of the six main planets and the ten satellites, not a single one moves, either in its forward motion or in its axial rotation, in any other direction than from west to east. Moreover, these directions are so precisely coordinated that they deviate only a little from a common plane, and this plane, to which everything is related, is the equatorial plane of the body which rotates on its axis at the central point of the entire system in exactly the same direction and which has become, through its predominant power of attraction, the reference point for all motions and thus necessarily participates in them as precisely as possible. (Kant, 1755, pg. 117)

Kant claimed this argument provided "proof that the collective movements arose and were determined in a mechanical way in accordance with general natural laws," (Kant, 1755, pg. 117) but he exaggerated the precision and consistency of these orbital parameters. Indeed, he was well aware of variations in the orbital inclination angle, and struggled to explain them. ${ }^{2}$ Nonetheless, Kant was highly confident for he had, as he next explained, disproven the null hypothesis (to use modern statistical parlance). To elucidate on the proof, Kant focused on the question of why the planets revolve about the sun in the same direction, for "it is clear that here there is no reason why the celestial bodies must organize their orbits precisely in one single direction, unless the mechanics of their development had determined the matter." (Kant, 1755, pg. 118) If they were arranged by the "immediate hand of God" then we would expect them to reveal deviations and differences.

Thus, God's choice would not have the slightest motive for tying them to one single arrangement, but would reveal itself with a greater freedom in all sorts of deviations and difference. (Kant, 1755, pg. 118)

In Kant, the random design argument is clear. The planets reveal a pattern rather than a random arrangement. Therefore, the planetary orbits must have originated not by the immediate hand of God but by natural processes. Kant

\footnotetext{
${ }^{2}$ Kant appealed to qualitative, ad hoc, explanations for variations in orbital parameters such as eccentricity and inclination. (Kant, 1755, pgs.48-49)
}

argued the solar system arose from a condensing cloud of particles. Kant was sure that the solar system arose via a mechanical sequence of natural laws. For Kant, these arguments demonstrated the reality of a mechanistic origin so clearly that we can entertain "no doubts about it." (Kant, 1755, pg. 117) This was proof that the solar system arose mechanistically in accordance with general natural laws.

In addition to Bernoulli and Kant, Buffon and Laplace proposed their own theories for the origin of the solar system. Buffon replaced Bernoulli's solar atmosphere idea with a comet that collides with the sun, spewing forth solar material that would later condense to form the planets. Buffon concluded that "It is therefore extremely probable, that the planets were originally parts of the sun." (Buffon, 1749, pg. 80)

Laplace replaced Buffon's idea with his Nebular Hypothesis. The Nebular Hypothesis called for a cloud of material about the sun that rotates and condenses to form the planets and sun. It was, claimed Laplace, the "true system of the world." (quoted in Brush, 1996, pg. 22) In spite of their theoretical differences, both Buffon and Laplace used the random design argument, with their own refined versions of Bernoulli's calculation, to support their confident conclusions. Buffon found that "By the doctrine of chances" the odds of such a coincidental alignment of the planetary inclination angles would be 7692624 to 1. (Buffon, 1749, pg. 65) Laplace made several calculations, eventually finding the odds of the solar system's patterns to be 537 million to 1 if they had arisen by chance:

Laplace was familiar with Newton's opinion that the regular motions of the planets proved their divine design. We know he was acquainted with Daniel Bernoulli's prize essay of 1734 on the subject, since in an earlier paper he had cited Bernoulli's method for calculating the probability that $n$ bodies all move in the same one of two possible directions if their motions are selected by chance: $2^{(-n+1)}$. In that paper Laplace had applied the method to six planets and ten satellites, finding the probabilities to be $2^{-15}=1 / 32768$. By 1796 he had made the coincidence even more unlikely by including the seventh planet, Uranus (discovered by William Herschel in 1781), as well as four more satellites, Saturn's rings, and the rotations of five planets, the Sun, the Moon, and one of Saturn's satellites (Iapetus). Thus of the 30 known motions in the Solar System, all are in the same direction. If these motions had been determined by chance, the probability that at least one of them would be different from the rest is extremely high $\left(1-2^{-29}\right)$. (Brush, 1996, pg. 21) 
In the ideas of Bernoulli, Kant, Buffon and Laplace the random design argument was key. They established a random design argument tradition, using what today is referred to as a null hypothesis to argue against design and arrive at what was virtually a proof of their respective contingency hypotheses. In this tradition, the hypotheses were underdetermined and in most cases opposing or even mutually exclusive. But confidence was extremely high, not from theoretical successes but from the clear refutation of the null hypothesis. Laplace could on the one hand be supremely confident and even assure Napoleon of his contingency theory (Brush, 1996, pg. 20) while, on the other hand, have nothing more than an underdetermined, untested, overarching notion of how the solar system actually arose. Indeed, Laplace failed to explain new observations such as the anomalous orbits of Uranus' moons, discovered by Herschel:

Laplace was aware when he first published his theory that Herschel had found the two satellites of Uranus to have orbits in a plane nearly perpendicular to the plane of the ecliptic. In 1798 Herschel announced that the satellites of Uranus have retrograde motion. While this amounted to only a slight revision of his earlier result - the orbit plane is still nearly perpendicular but is tilted in the other direction - it was still [Herschel explained] "a remarkable instance of the great variety that takes place among the movements of the heavenly bodies" since previously all known motions took place in the same direction. (Brush, 1996, pg. 21)

This was a remarkable variation, and it highlights a crucial aspect of this random design reasoning. The use of what we refer to today as null hypothesis testing allowed one to obviate the scientific activity of developing and deriving models, mathematical relationships, and mechanisms. Instead, a dichotomy is drawn: either the design of the solar system is random, or else the solar system arose by a yet to be determined contingency. The alternate hypothesis does not specify any particular mechanism, that is the subject of after-the-fact theorizing.

Various mechanisms can be hypothesized or dropped, as needed. As we have seen, Bernoulli, Buffon, Kant, and Laplace each presented different mechanisms with complete confidence. Investigations of possible mechanisms would be viewed as a sign of scientific progress, no matter how often they turn out to be flawed. Meanwhile anomalies, such as the two satellites of Uranus with perpendicular orbits, would be viewed as curiosities and grounds for further scientific research. They do no harm to the underlying null hypothesis rejection, for the most they can do is slightly nudge the statistics. Furthermore, such anomalies can simply be ignored in the null hypothesis calculation, as Laplace did. (Brush, 1996, pg. 22) This approach of rejecting the random design null hypothesis made the science more of a statistical exercise than of providing specific and falsifiable models and mechanisms to adequately explain natural phenomena. To summarize these eighteenth century theories of the origin of the solar system, these theories included or promoted the following six important elements for our purposes.

1. The random design argument is not limited to objects, but also applies to design parameters. In this case, it was not the celestial bodies such as planets and moons themselves that were of concern so much as their orbital parameters. The direction of their revolutions about the sun, the direction of their spin, and the inclination angle of their orbits about the sun were of concern. Newton referenced these parameters in his design arguments and now they had become subject to random design testing.

2. Nature should present a full continuum of values of these orbital design parameters. Those values should randomly fill the design space. The motivation here was from pattern, not mechanism. In other words, these new theories for the origin of the solar system were not motivated by the finding of a compelling, explanatory mechanism, but rather by the patterns found within the solar system structure.

3. This new tradition, based on the random design argument, produced highly underdetermined theories. Whereas Newtonian physics could claim a rather tight, mathematical, cause and effect relationship between forces and motion, this new tradition produced broad, phenomenological theories which did not provide detailed descriptions of the outcomes. While this is to be expected of such phenomenological hypotheses they, in fact, did not even account for the very observables, such as the planetary inclination angles, of concern to this tradition. That is, the different hypotheses of Bernoulli, Buffon, Kant, and Laplace, did not provide specific forces or causes that would result in, or explain, the observed variation in inclination angles, in any sort of direct sense. The solar atmosphere, a comet striking the sun, and a condensing cloud were meant to explain the common design parameters, but they did not provide any specific causes that would produce the variation in inclinations angles of the planetary orbits.

4. The contingency mechanisms were unrelated to the random design reasoning that had underwritten them. Bernoulli's solar atmosphere hypothesis, for example, 
did not derive in any way from his statistical calculations. The same is true for Buffon's, Kant's and Laplace's hypotheses. This new tradition consisted of a confirmation step (the falsification of the random design null hypothesis) which was entirely disjoint from the derivation of the mechanism. By falsifying design, the scientist was free to speculate about the mechanism, in spite of theoretical problems.

5. In spite of the theoretical shortcomings, and the dramatic differences between the different theories, Bernoulli, Buffon, Kant, and Laplace each had the utmost confidence in the veracity of their respective theories. Each of these theoreticians produced high-level, phenomenological theories with theoretical shortcomings. And each lacked theoretical details. Looking back, it seems obvious that each theory was merely one of several possibilities. Indeed, Stephen Brush has pointed out that those early hypotheses, as well as those from the following centuries, fell into two broad categories: monistic and dualistic. Monistic theories describe the sun and planets as originating from a common phenomenon (e.g., a condensing cloud) whereas dualistic theories have them originating from distinct, separate phenomena (e.g., the sun forms first, and later a comet strike produces the planets). Brush points out the solar system origin problem remains unsolved simply because there has continued to be a switching in preference between these two mutually exclusive categories. (Brush, 1996, pg. 4) But the high confidence of Bernoulli, Buffon, Kant, and Laplace did not arise from any theoretical success. It is not as though they had produced highly detailed theories that convincingly explained what we observe. Instead, their high confidence was a result of the random design tradition.

6. The spectacular failure of the random design null hypothesis had a significant impact on the philosophy of science within this tradition. Given that it was interpreted as an undeniable proof against design of the solar system, it relieved scrutiny of the scientific theories for its origin. Bernoulli, Buffon, Kant, and Laplace could proclaim full confidence in their respective theories with little or no demonstration that their mechanisms actually could, in fact, create the solar system. And shortcomings in their mechanisms - whether known at the time or discovered later - were viewed more as interesting research problems rather than evidences against the theory. This made the theories more robust to scientific failures and resistant to falsification. Orbits that violated the expected pattern were of little concern. They could always be explained by contingent events, something which Newton himself called upon to explain the variations in the inclination an- gles of the planetary orbits. If the choice was between contingent events and random design, then contingent events were the obvious preference since the random design null hypothesis had been shown to be false. So the spectacular failure of the null hypothesis served to underwrite substantial speculation in science. Explanations could be fairly vague and failures could be accommodated as research problems.

These theories of the origin of the solar system are important for their influence on eighteenth century thought but, as we shall see next, they are also important because this new philosophy of science would emerge in the confirmation of biological theories of origin, particularly in the twentieth century.

\section{The influence of the random design argument in biological evolution}

In advancing his theory of evolution Charles Darwin made a wide range of arguments. Many of those arguments referred to patterns in the biological world which, Darwin argued, would not be expected under independent creation. Indeed, one of his strong arguments was from the mere existence of similarities between the different species which he summarized in Chapter 13 of Origin:

What can be more curious than that the hand of a man, formed for grasping, that of a mole for digging, the leg of the horse, the paddle of the porpoise, and the wing of the bat, should all be constructed on the same pattern, and should include the same bones, in the same relative positions? (Darwin, 1859, pg. 434)

Darwin's rhetorical question highlights the role of the random design argument in this interpretation of the evidence. There should be no such obvious pattern. Instead, these anatomical designs should fill the design space:

We never find, for instance, the bones of the arm and forearm, or of the thigh and leg, transposed. Hence the same names can be given to the homologous bones in widely different animals. (Darwin, 1859, pg. 434)

Darwin found these seemingly arbitrary patterns throughout biology: 
We see the same great law in the construction of the mouths of insects: what can be more different than the immensely long spiral proboscis of a sphinx-moth, the curious folded one of a bee or bug, and the great jaws of a beetle? - yet all these organs, serving for such different purposes, are formed by infinitely numerous modifications of an upper lip, mandibles, and two pairs of maxillæ. Analogous laws govern the construction of the mouths and limbs of crustaceans. So it is with the flowers of plants. (Darwin, 1859, pgs. 434435)

As with the shared inclination angles of the planetary orbits, these shared features could not be explained "by utility or by the doctrine of final causes." (Darwin, 1859, pg. 435) In other words, Darwin argued the design space was largely unconstrained. Other designs were possible.

It is a testament to the acceptance of random design thinking that Darwin could so casually suggest reversing "the bones of the arm and forearm, or of the thigh and leg," as though such wholesale restructuring would have no functional effects. Darwin could not know that the bones of the arm and forearm, or of the thigh and leg, could be transposed with no loss of utility. For our purposes here, the point is that the expectation that nature ought to fill the design space parallels the eighteenth century random design interpretation of cosmology, this time applied to the biological world.

After Darwin this tradition was taken for granted. Here is how Mark Ridley explains this argument, updated to include the universal genetic code:

Homologous similarities between species provide the most widespread class of evidence that living and fossil species have evolved from a common ancestor. The anatomy, biochemistry, and embryonic development of each species contains innumerable characters like the pentadactyl limb and the genetic code: characters that are similar between species, but would not be if the species had independent origins. (Ridley, 1993, pgs. 48-49)

Similarly, Jerry Coyne restates this idea that the anatomies of different species should share no common patterns and should be unique:

There is no reason why a celestial designer, fashioning organisms from scratch like an architect designs buildings, should make new species by remodeling the features of existing ones. Each species could be constructed from the ground up. (Coyne, 2009, pg. 54)

This argument from similarities across the species is ubiquitous and appears in a variety of contexts. Whales are mammals and, as this example points out, would be unlikely to sport mammalian characteristics under the random design hypothesis. Clearly there is a pattern at work:

The probability of mammalian characteristics (such as having hair and feeding their young with milk, as well as a number of defining skeletal characteristics) arising in a separate, unrelated lineage is a pretty big stretch. (Venema and McKnight, 2017, pg. 14)

It is "a pretty big stretch" because the expectation is random design. Likewise, the fossil record should be random rather than exhibiting patterns: "So the appearance of species through time, as seen in the fossils, is far from random." (Coyne, 2009, pg. 29) These are longstanding, powerful arguments for evolutionary contingency, based on the failure of random design in nature.

These qualitative interpretations of the biological world have been augmented with more quantitative arguments. With the formalization of statistical testing, more data, and more computational power, the random design argument has been incorporated into biological studies in more sophisticated and subtle ways. The remainder of this section presents three such cases.

\subsection{Protein sequences and evolutionary trees}

In 1982 David Penny and co-workers presented influential arguments for evolution that fall squarely within the random design argument tradition. Penny used five proteins (cytochrome $\mathrm{C}$, hemoglobin $\mathrm{A}$, hemoglobin $\mathrm{B}$, fibrinopeptide A and fibrinopeptide $\mathrm{B}$ ) to infer the evolutionary relationships between eleven different species (rhesus monkey, sheep, horse, kangaroo, mouse, rabbit, dog, pig, human, cow, and ape). (Penny, Foulds, and Hendy, 1982) Just as visible features, such as the heart, vary from species to species, so too proteins, such as hemoglobin A, vary from species to species in their amino acid sequence. The Penny et. al. random design null hypothesis stated that the protein relationships between species are random. For example, the cytochrome $\mathrm{C}$ protein is highly similar between the 
human and ape. According to the null hypothesis, this tells us nothing about how the other four proteins compare between the human and ape.

Of course this random design null hypothesis fails spectacularly. Protein differences between species tend to be correlated. Penny et. al. expressed this failure quantitatively using evolutionary trees. First, they used the five proteins, one at a time, to construct five different evolutionary trees. According to the random design null hypothesis, these trees should bear no particular similarity. The evolutionary tree derived by comparing one protein across the different species should bear no relation to the evolutionary tree derived by comparing a second protein across those species. The protein sequence differences should be random and so the resulting evolutionary trees that are computed, from those differences, are also randomly related, according to the null hypothesis.

Of course this was not true. Each of the five proteins, independently, produced similar trees. The different evolutionary trees, based on the different proteins, did show some significant differences. One of the trees showed the dog relatively far from the human (9 species distant out of a possible 10) whereas others show the dog relatively close to the human (3 species distant out of 10). And the same is true for the mouse.

Yet the results were far from a random sampling of the space of all possible evolutionary trees. The null hypothesis was clearly false, and the paper concluded, "There is thus a strong divergence away from random towards the trees being very similar." (Penny, Foulds, and Hendy, 1982, pg. 200) For Penny this provided "strong support" for the theory of evolution. A follow up study found that with those eleven different species, "the probability of randomly selecting trees this similar is $5.37 \times 10^{5}$." (Penny and Hendy, 1986, pg. 408) The null hypothesis was falsified and the trees "were much more similar than would be expected by chance." (Penny and Hendy, 1986, pg. 403) As one textbook explained, "The key observation was that the trees for all five proteins are very similar," and there is no reason for this if the species were "separately created." (Ridley, 1993, pg. 52) This example illustrates the importance of the random design argument in the evaluation and confirmation of evolution. The random design tradition has become a textbook norm.

In a later 2013 study Penny and co-workers used an updated approach with more data and even more powerful results were obtained: "Combining results for all 51 genes gives a $P$ value for our non-evolutionary null model of $\approx 2 \times 10^{19}$." (White, Zhong, and Penny, 2013) That result was for one of their eight data sets. Combining all eight data sets resulted in a $P$ value of $10^{132}$ :

Even on our conservative test, the probability that chance could produce the observed levels of ancestral convergence for just one of the eight datasets of 51 proteins is $\approx 1 \times 10^{19}$ and combined over 8 datasets is $\approx 1 \times 10^{132}$. (White 2013)

The chance null hypothesis was clearly false and it seemed that evolution and common ancestry were undeniable:

By comparison, there are about $10^{80}$ protons in the universe, hence the probability that the sequences could have been produced by a process involving unrelated ancestral sequences is about $10^{50}$ lower than picking, among all protons, the same proton at random twice in a row. (White 2013)

According to this random design argument, the molecular differences, across different species are expected to fill the design space at random. This is the null hypothesis and it is extremely unlikely given the patterns in the molecular sequence data. Given the failure of the null hypothesis, the alternate hypothesis is taken to be true. As one textbook concluded, referring to Penny, Foulds, and Hendy (1982):

Penny et. al. suggested that this pattern is so compelling that the [null] hypothesis of separate ancestry should be emphatically rejected as being incompatible with the data. While science rarely deals with certainty, it is fair to say that evolution from common ancestry is now supported beyond any reasonable doubt. (Baum and Smith, 2012, pg. 23)

Thus the random design argument has not only provided a null hypothesis for use in justifying evolutionary theories, it has done so in striking fashion. The assumption that molecular sequences should exhibit random variations has produced astronomical improbabilities and high confidence in the alternate hypothesis; namely, evolution.

\subsection{Systematics}

It has been known since antiquity that the species fall into groupings and in the eighteenth century Linnaeus used a hierarchical structure to organize the species. Species were 
grouped into genera, genera were grouped into orders, orders were grouped into classes, and classes were grouped into kingdoms. This nonrandom pattern was yet another failure of random design, and so another argument for evolution:

The several subordinate groups in any class cannot be ranked in a single file, but seem rather to be clustered round points, and these round other points, and so on in almost endless cycles. On the view that each species has been independently created, I can see no explanation of this great fact in the classification of all organic beings. (Darwin, 1859, pgs. 128-129)

In recent decades this taxonomic failure of the random design null hypothesis has been quantified for many different sets of species and characters. The consistency index (CI), for example, measures the degree of consistency between (i) a set of character data, from a set of species and (ii) the tree used to describe their evolutionary history. For example, consider two groups of species that are distant on the evolutionary tree, and yet one of the species, in one of the groups, surprisingly shares a few of its character states with the species in the other group. These type of inconsistencies, sometimes striking, are not uncommon in biology (Morris, 2004), and CI measures how prevalent they are in a data set. CI ranges from $0-1$, with a value of 1 indicating an entirely consistent data set, and a value of 0 indicating an entirely inconsistent data set. Note that even randomized character data have some consistencies just by chance, and so produce CI values greater than 0 .

As a test of evolution and common ancestry, a random $\mathrm{CI}$ measure is used as the null hypothesis. In other words, the character data are first randomized, and then the CI value is computed. This process is repeated many times to establish the distribution of CI values that can be expected in the randomized case. The real CI values (based on the actual character data) are often far from 1 due to inconsistencies between the species. In fact, the real CI values are often closer to the randomized CI value than to 1 . But nonetheless, the real CI values are outside the range of randomized CI values. (Archie, 1989; Faith and Cranston, 1991) In other words, though far from 1, the real CI values do not fall within the typical range of randomized values. The null hypothesis can be rejected, and this is cited as powerful evidence for the evolution and common ancestry of the species. The strength of the evidence rests not on the absolute CI values, and proximity to a value of 1 , but rather on the CI values relative to their respective randomized values:

When we sequence the same gene from multiple species and subject it to this statistical [CI] test, we generally find huge differences between the observed CI and the [randomized] CI predicted under separate ancestry. (Baum and Smith, 2012, pg. 21)

For example, Salamandridae is a family of salamanders consisting of true salamanders and newts. Data on 40 characters from these species (Wake and Ozeti, 1969) yielded a relatively low CI value of .596. When the data were randomized, ${ }^{3}$ however, the CI value ranged from .422-.463. Though far from 1 , the actual CI value was well outside of the range of values resulting from randomized data, and thus interpreted as powerful evidence for evolution and common ancestry.

\subsection{Evolution of primates}

My final example of the influence of the random design argument in the biological sciences is a systematic study of the evidence for common ancestry within the primate species. (Baum, 2016) This study gathered several different, large scale, data sets, including molecular, morphological, and biogeographical data. These data were then used in several different tests of common ancestry, using separate ancestry as the null hypothesis. As in the previous examples, the general approach is to construct a model of the null hypothesis, compare the empirical data to the null hypothesis, and compute the $P$ value to determine if the null hypothesis can be rejected. Exceedingly small $P$ values indicate that the null hypothesis (separate ancestry) can be rejected, and so the alternate hypothesis (common ancestry) is accepted. The study performed tests both at the species and the family level. Figure 1 summarizes the five species-level tests.

As in our earlier examples, in each of these tests the null hypothesis expects no pattern. It represents what would result from a randomized version of the observed data. A typical technique, as described in Section 3.2, is to rearrange the character state data so they are randomized for each species.

\footnotetext{
${ }^{3}$ The randomization process is as follows. For each of the 40 characters, there is a character state which is observed for each species. These data can be arranged in a matrix where each row represents a species, and each column represents a character. In the randomization process the character state data in each column are moved to a randomly selected row. Therefore, the set of character state data in each column is not changed, but the data are rearranged, as they are randomized for each species.
} 
Figure 1: Summary of the five species-level tests used to reject the null hypothesis of separate ances-

try

\begin{tabular}{|l|l|}
\hline Test name & Null hypothesis procedure \\
\hline Agreement within a Single Data Matrix & $\begin{array}{l}\text { Randomize character state data and measure pattern similarity } \\
\text { (consistency) between the different characters. }\end{array}$ \\
\hline $\begin{array}{l}\text { Phylogenetic Autocorrelation in a Single Char- } \\
\text { acter }\end{array}$ & $\begin{array}{l}\text { Randomize character state data and compare with the } \\
\text { evolutionary tree derived from DNA sequences. }\end{array}$ \\
\hline $\begin{array}{l}\text { Testing for Autocorrelation in the Presence of } \\
\text { Potential Covariates }\end{array}$ & $\begin{array}{l}\text { Randomize character state data and focus on two characters. } \\
\text { Assume that other characters are independent, and so do not help } \\
\text { to explain how the character compares with the evolutionary tree } \\
\text { derived from DNA sequences. }\end{array}$ \\
\hline $\begin{array}{l}\text { Agreement between the Trees Estimated from } \\
\text { Different Datasets }\end{array}$ & $\begin{array}{l}\text { Updated version of Penny, et. al., 1982 test, described above in } \\
\text { Section 3.1 Compute the difference (or distance) between } \\
\text { evolutionary trees, each derived from a single gene, using } \\
\text { evolutionary trees that are randomly generated for each gene, for } \\
\text { the species in question. This is equivalent to deriving the } \\
\text { evolutionary trees using randomized gene sequences. }\end{array}$ \\
\hline Deficit of Derived Character States in Fossils & $\begin{array}{l}\text { For both extant and extinct species, measure the distance to the } \\
\text { root of the evolutionary tree. On average, the null hypothesis } \\
\text { expects no difference. }\end{array}$ \\
\hline
\end{tabular}


Some of these tests were run repeatedly with different data sets, resulting in more than merely five $P$ value results. And as in our earlier examples, the computed $P$ values for most of the tests were exceedingly small. This was true for the tests run at the family level as well. For example, many of the $P$ values were less than $10^{--300}$. Given these infinitesimal $P$ values, the authors overwhelmingly rejected the separate ancestry null hypothesis. Following Penny, the authors explained that for several of the tests the separate ancestry null hypothesis was less likely than the probability of picking the correct atom at random among the estimated $10^{80}$ atoms in the known universe.

Echoing Daniel Bernoulli and the eighteenth century cosmological evolutionary theories discussed in Section 2, the authors explained the essence of this approach. Even a single character can provide strong evidence for common ancestry if its observed states, in different species, is a small subset of its possible states:

in principle, a single characteristic that could exist in very many alternative states can provide evidence of [common ancestry] when the states seen in a clade, or life as a whole, occupy a smaller subset of state space than would be expected under [separate ancestry]. (Baum, 2016, pg. 1362)

This is the random design argument. If there is no reason to think the character state could not take on "very many alternative states," and yet it occupies "a smaller subset of state space," then the null hypothesis can be rejected. Just as the planetary inclination angles occupied a small subset of the possible values and so proved cosmological evolution, so too biological characters that occupy a small subset of the possible values prove biological evolution. The authors suggest this random design philosophy of science may find broader application in the historical sciences:

By looking across different historical sciences and the sources of evidence that they use in support of accepted claims, we may hope that a general theory of statistical history may emerge. Thus, we hope that this article will not just stimulate further philosophical and statistical research on evidence for [common ancestry], but also on broader questions in historical inference. (Baum, 2016, pg. 1362)

\section{Conclusions}

The random design argument is an important tradition in the history of science. The fundamental idea is that nature's designs should fill the design space, otherwise contingency is implicated. Over the past three centuries the random design argument, and in particular testing of the random design null hypothesis, has provided for an extremely powerful philosophy of science. It is a solution to the ageold difficulty of how to prove a scientific theory. If a null hypothesis can be constructed which complements the theory in question (the alternative), such that the two theories are the only two possible explanations, then falsifying the null hypothesis is equivalent to proving the alternative. If the null hypothesis has a probability of less than $10^{-10}$ then the alternative certainly qualifies as a "fact," by any reasonable understanding of that term. This paper shows how the random design argument has motivated this philosophy of science. The following five points summarize this move.

1. God, if He was directly responsible for creation, should fill the design space. Objects within nature, and their design parameters, have a range of possibilities, and nature should fill that capacity. Genuine possibilities should not go unfulfilled. This is the null hypothesis.

2. The null hypothesis, by definition, complements the alternate hypothesis. The null hypothesis and the alternate hypothesis span the space of possible hypotheses. They are mutually exclusive and there are no other possible explanations.

3. Rejection of the null hypothesis implies that the alternate hypothesis is a fact. Contingency, both in cosmology and biology, has been proven to be a fact by the failure of the respective null hypotheses.

4. This philosophy of science obviates mechanism. The alternate hypothesis can be proven to be true even though it lacks specific or detailed mechanisms. The development and testing of the scientific mechanisms can come later and can sustain many failures. Indeed, mechanisms that are proposed typically do not even explain the very data used to reject the null hypothesis. Variations in the planetary inclination angles, or variations in the species hierarchical structure, are not explained by the condensing nebula or common ancestry hypotheses, respectively. Additional, ad hoc, contingent events and causes are required.

5. This philosophy of science, with its rejection of the null hypothesis, leads to exceptionally high confidence. The alternate (i.e., contingency) hypothesis simply must be 
true, in spite of ambiguities, lack of specifics, or failures.

The validity of the statistical reasoning behind these five points is dependent on one special property of the null hypothesis. As stated above, the null hypothesis must be the complement of the alternate (contingency) hypothesis. There can be no overlap and no gap between the two hypotheses. This is crucial and its impact to the validity of the reasoning should not be underestimated. The power of this philosophy of science lies in its ability to (i) craft relatively simple, tractable null hypotheses which can be evaluated and rejected, while (ii) credibly maintaining the premise that it is a legitimate null hypothesis; that is, that it is the complement of the alternate hypothesis.

The assumption that if the planetary inclination angles, or the species' characters, do not fully populate the possible range of values, then they must have arisen via contingent events, is underwritten by an underlying theological assumption, not by empirical science. This is an example of how a nonscientific idea influences not merely theory development, but theory evaluation and even theory confirmation. The origin and details of this theological assumption are beyond the scope of this paper.

In the past century historians have increasingly demonstrated how nonscientific influences have played a role in the development of scientific theories. Less attention has been paid to the influence of nonscientific ideas on theory evaluation. Why are certain theories held with such high confidence? How are they known to be facts? This paper has explored one such example of how the random design argument has contributed to the high confidence in some scientific theories, and how that confidence relies on theological assumptions. From the perspective of empirical science, such confidence is not warranted.

\section{References}

Archie, J W (1989). "A randomization test for phylogenetic information from systematic data". In: Systematic Zoology 38, pp. 239-252.

Baum, D et al. (2016). "Statistical evidence for common ancestry: Application to primates". In: Evolution 70, pp. $1354-1363$.

Baum, D and S Smith (2012). Tree Thinking: An Introduction to Phylogenetic Biology. New York: W. H. Freeman.
Bernoulli, D (1734). "Physical and Astronomical Researches on the Problem Proposed for the Second Time by the Academie Royale des Sciences de Paris". In: Recueil des pièces qui ont remporté les prix de l'Académie royale des sciences, vol. 3. Trans. by Richard J. Pulskamp. Paris: Jombert. URL: http://cerebro.xu.edu/math/ Sources / DanBernoulli / 1734\% 5C_planets $\% 20$ and $\%$ 20comets.pdf.

Brush, S (1996). Nebulous Earth: The Origin of the Solar System and the Core of the Earth from Laplace to Jeffreys. Cambridge: Cambridge University Press.

Buffon, G L C (1749). "Of the Formation of Planets". Trans. by William Smellie. In: Natural History: General and Particular 1.1.

Coyne, J (2009). Why Evolution is True. New York: Viking.

Danielson, D (2000). The Book of the Cosmos. New York: Basic Books.

Darwin, C (1859). The Origin of Species. London: John Murray.

Faith, D and P Cranston (1991). "Could a cladogram this short have arisen by chance alone - on permutation tests for cladistic structure". In: Cladistics 7, pp. 128.

Fisher, R (1935). The Design of Experiments. Edinburgh: Oliver and Boyd.

Hunter, C (2007). Science's Blind Spot. Grand Rapids: Brazos.

Hunter, C (2014). "Darwin's Principle: The Use of Contrastive Reasoning in the Confirmation of Evolution". In: HOPOS 4, pp. 106-149.

Kant, I (1755). Allgemeine Naturgeschichte und Theorie Des Himmels /Universal natural history and theory of heaven]. Trans. by Ian Johnston. Arlington, VA: Richer Resources.

Manuel, F (1968). A Portrait of Isaac Newton. Cambridge, MA: Belknap.

Morris, S C (2004). Life's Solution: Inevitable Humans in a Lonely Universe. Cambridge: Cambridge University Press.

Newton, I (1687). The Principia. Trans. by Andrew Motte. New York: Prometheus Books.

Newton, I (1718). Opticks. London. URL: http : / / www . newtonproject . sussex . ac . uk / view / texts / normalized/NATP00051.

Penny, D, L Foulds, and M Hendy (1982). "Testing the theory of evolution by comparing phylogenetic trees constructed from five different protein sequences". In: $\mathrm{Na}$ ture 297, pp. 197-200.

Penny, D and M Hendy (1986). "Estimating the reliability of evolutionary trees". In: Molecular and Biological Evolution 3, pp. 403-417.

Ridley, M (1993). Evolution. Boston: Blackwell. 
Rogers, A (2011). The Evidence for Evolution. Chicago: University of Chicago Press.

Shea, W (1986). "Filled with Wonder: Kant's Cosmological Essay, the Universal Natural History and Theory of the Heavens". In: Kant's Philosophy of Physical Science. Ed. by R E Butts. Dordrecht: D. Reidel.

Venema, D and S McKnight (2017). Adam and the Genome: Reading Scripture After Genomic Science. Grand Rapids: Brazos.

Wake, D and N Ozeti (1969). "Evolutionary Relationships in the Family Salamandridae". In: Copeia 1969, pp. 124-137.

White, W T J, B Zhong, and D Penny (2013). "Beyond Reasonable Doubt: Evolution from DNA Sequences". In: PLoS ONE 8 (e69924). 